\title{
Cassini observations of planetary-period oscillations of Saturn's magnetopause
}

\author{
K. E. Clarke, ${ }^{1}$ N. André, ${ }^{2}$ D. J. Andrews, ${ }^{1}$ A. J. Coates,${ }^{3}$ S. W. H. Cowley, ${ }^{1}$ \\ M. K. Dougherty, ${ }^{4}$ G. R. Lewis, ${ }^{3}$ H. J. McAndrews, ${ }^{3}$ J. D. Nichols, ${ }^{1}$ T. R. Robinson, ${ }^{1}$ \\ and D. M. Wright ${ }^{1}$
}

Received 9 August 2006; revised 19 October 2006; accepted 6 November 2006; published 7 December 2006.

[1] Examination of Cassini magnetic field and plasma data in the outer boundary regions of Saturn's magnetosphere shows that magnetopause oscillations at the planetary period commonly occur, in phase with plasma pressure variations inside the magnetosphere. The peak-to-trough amplitude of the boundary oscillations mapped to the planet-Sun line is estimated to be typically $\sim 2 \mathrm{R}_{\mathrm{S}}$, corresponding to a $\sim 10 \%$ change in the boundary radius. The change in internal pressure required to produce such motions is estimated to be $\sim 40 \%$ of the background values. A qualitative physical picture is proposed in which a compressive wave propagates outward through the sub-corotating outer magnetospheric plasma, originating from a corotating source in the nearer-planet region. Citation: Clarke, K. E., et al. (2006), Cassini observations of planetary-period oscillations of Saturn's magnetopause, Geophys. Res. Lett., 33, L23104, doi:10.1029/2006GL027821.

\section{Introduction}

[2] The dynamic pressure of the solar wind is a primary parameter governing the location of the magnetopause boundary of a planetary magnetosphere. Empirical models of the boundary position parameterized by this quantity have been presented for Jupiter by Huddleston et al. [1998] and Joy et al. [2002] and for Saturn by Slavin et al. [1983] and Arridge et al. [2006], the latter being based on recent data from the Cassini orbiter space mission. In the case of the Earth, the direction of the interplanetary magnetic field is also known to play a role, due to the erosion of closed flux from the dayside boundary by reconnection [e.g., Shue et al., 1997], and indications of a similar effect have also been found at Jupiter [Kivelson and Southwood, 2003]. In the case of Saturn's magnetosphere, however, Espinosa et al. [2003a] also presented evidence for internal control of the boundary, showing that a sequence of magnetopause crossings took place on the outbound pass of the Pioneer-11 spacecraft near the dawn meridian that were separated by roughly the planetary rotation period of $\sim 10.75 \mathrm{~h}$. Magnetic perturbations with this period were also observed inside the

\footnotetext{
${ }^{1}$ Department of Physics and Astronomy, University of Leicester, Leicester, UK.

${ }^{2}$ Research and Scientific Support Department, European Space Agency, Noordwijk, Netherlands.

${ }^{3}$ Mullard Space Science Laboratory, University College London, Dorking, UK.

${ }^{4}$ Blackett Laboratory, Imperial College London, London, UK.
}

Copyright 2006 by the American Geophysical Union. 0094-8276/06/2006GL027821 magnetosphere during the fly-bys of the Pioneer and Voyager spacecraft. Espinosa et al. [2003b] hypothesized that a compressive wave is launched into Saturn's magnetosphere by some corotating anomaly at the planet, yet to be detected directly, leading to magnetic oscillations at the planetary period within the magnetosphere, and to corresponding motions of the boundary. Subsequent Cassini observations have shown that these oscillations are ubiquitous inside Saturn's magnetosphere, and that their observed period undergoes modest Doppler shifts due to spacecraft motion through the outward-propagating 'spiral' wave field [Cowley et al., 2006]. Here we examine Cassini boundary data that correspondingly show numerous examples of multiple boundary crossings separated approximately by the planetary period, thus uniquely demonstrating for Saturn clear internal control of the boundary location additional to that exerted by the interplanetary medium. In this paper we show some examples of this behavior, and discuss their implications.

\section{Data}

[3] In this section we exemplify the evidence for magnetopause boundary oscillations in Cassini data by presenting three successive boundary region passes, specifically the inbound and outbound passes of Rev 16 and the inbound pass of Rev 17. We use magnetic field data from the fluxgate sensor of the magnetic field investigation [Dougherty et al., 2004], together with electron data from the electron spectrometer (ELS) sensor of the CAPS instrument [Young et al., 2004].

[4] Figure 1 shows data for Rev 16, covering the interval from day 281 to day 290 (8-17 October), inclusive, of 2005. The top panel is a spectrogram of the electron flux over the energy range 0.58 to $26040 \mathrm{eV}$ for ELS anode 5, color-coded according to the scale on the right hand side. Note that at radial distances beyond $\sim 10 \mathrm{R}_{\mathrm{S}}$ the intense fluxes below a few eV are spacecraft photoelectrons. The next three panels show the spherical polar radial $(r)$, colatitudinal $(\theta)$, and azimuthal $(\varphi)$ components of the magnetic field with respect to the planet's spin/magnetic axis, with the 'Cassini' internal field model subtracted [Dougherty et al., 2005]. In the bottom panel the blue data show the magnetic field pressure on a logarithmic scale. The internal field has not been subtracted in this case, and is indicated by the green dashed line. Field magnitudes may be read from the scale on the right-hand side. The red line in this panel shows the electron pressure derived from the ELS distributions corrected for spacecraft potential, thus eliminating the photoelectron contribution. Ion pressures are not 


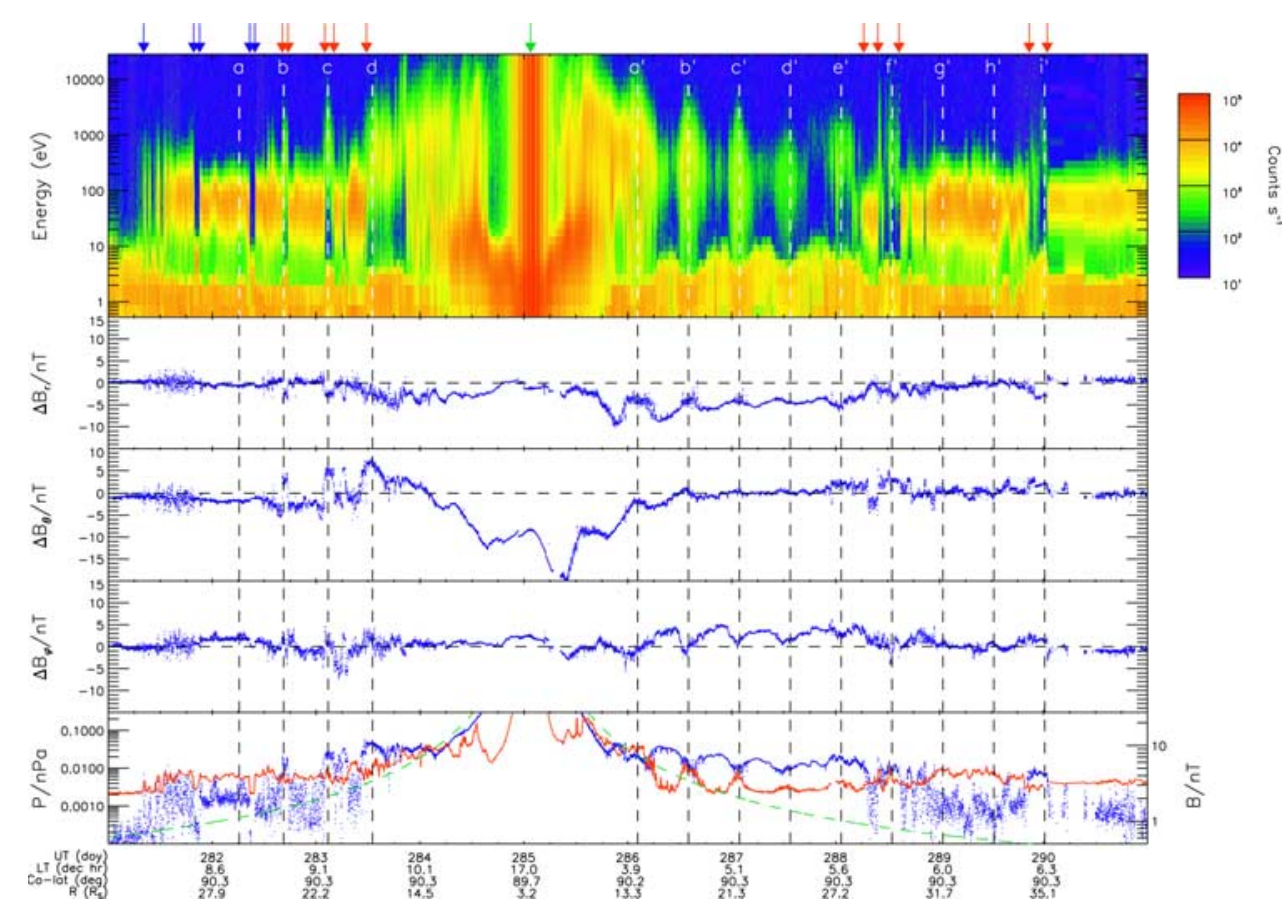

Figure 1. Electron flux spectrogram and magnetic field data from Cassini Rev 16, days 281-290.

routinely available for this interval, but in the middle and outer magnetosphere are found typically to be higher than electron pressures by factors of $2-3$ (M. F. Thomsen, personal communication, 2006). At the top of Figure 1, principal bow shock and magnetopause crossings are indicated by the blue and red arrows, respectively, while the green arrow indicates periapsis. At the bottom of Figure 1 we provide spacecraft positional information, specifically local time, colatitude (again with respect to the spin/magnetic axis), and radial distance from the center of the planet. It can be seen that Cassini was located very close to Saturn's equatorial plane throughout the interval (colatitude $\sim 90^{\circ}$ ).

[5] The orbit is also depicted in Figure 2, projected onto Saturn's equatorial $(\mathrm{X}-\mathrm{Y})$ plane with the Sun to the left. The $\mathrm{Z}$ axis is thus directed along the spin/magnetic axis, the $\mathrm{X}-\mathrm{Z}$ plane contains the Sun, and Y points towards dusk, completing the orthogonal right-handed triad. The plot covers an interval from one day before to one day after that shown in Figure 1, with the dots on the trajectory marking the start of the days numbered. At the start of day 281 the spacecraft was inbound in the mid-morning sector at an initial radial distance of $\sim 32.3 \mathrm{R}_{\mathrm{S}}$. It underwent periapsis passage at $\sim 3.0 \mathrm{R}_{\mathrm{S}}$ near the beginning of day 285 , and then passed outbound through the magnetosphere in the dawn sector, reaching a radial distance of $\sim 37.6 \mathrm{R}_{\mathrm{S}}$ at the end of day 290. The dashed lines in Figure 2 are Arridge et al. [2006] model magnetopauses, the outer one corresponding to a solar wind dynamic pressure of $0.01 \mathrm{nPa}$, the inner one to $0.1 \mathrm{nPa}$, spanning the usual range of values at Saturn. The circled dots on the trajectory mark the locations of the last inbound and the first outbound magnetopause crossings observed, thus indicating a relatively high solar wind dynamic pressure during the orbit and a relatively compressed magnetosphere.

[6] We focus first on the outbound pass of Rev 16, starting on day 286. Examining the magnetic field data, we see a sequence of four oscillations in field strength and direction on days 286 and 287 (labeled $a^{\prime}$ to $d^{\prime}$ ), of the form discussed previously by Espinosa et al. [2003a] and Cowley et al. [2006]. These oscillations principally involve the radial and azimuthal components, with the colatitudinal component remaining close to zero after the middle of day 286. Looking at the electron spectrogram, we find corresponding oscillations in the electron data over essentially the full range of energies sampled, with maxima in the flux occurring at approximately the same time as minima in the field strength, such that the field and electron pressures vary in anti-phase. In the first three of these flux maxima the electron pressure reached values comparable with the field pressure, which, with the ion pressure added, indicates that $\beta \geq 1$ conditions occurred at these times ( $\beta$ being the ratio of the total plasma pressure to the magnetic pressure). We note that the magnetic field was strongly disturbed during these intervals, characteristic of $\beta \geq 1$, while in the regions

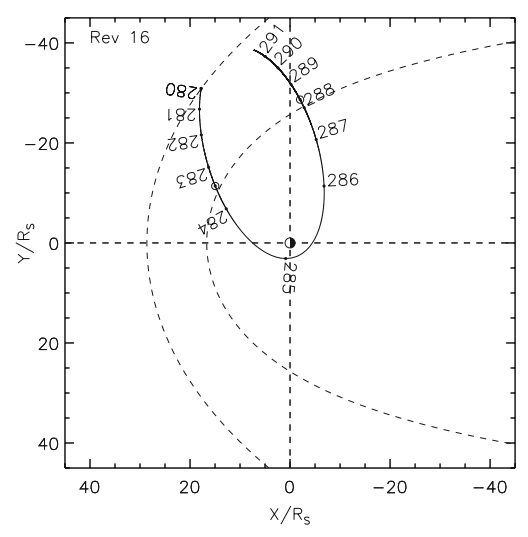

Figure 2. Orbit plot for Cassini Rev 16, days 280-291 inclusive. 
between where the electron pressures were much smaller than the field pressures, the field was more smoothly varying, characteristic of low $\beta$ conditions. Using the combined field and plasma data we are able to determine the period of the oscillations, and find an essentially constant period of $\sim 11.75 \mathrm{~h}$, as indicated by the vertical dashed lines which approximately mark electron flux maxima and field strength minima. This period is significantly longer than the planetary period of $\sim 10.75 \mathrm{~h}$ [Giampieri et al., 2006], due principally to the Doppler shift resulting from the spacecraft's radial motion through the wave field [Cowley et al., 2006]. The radial wave phase speed implied by the Doppler shift is $\sim 50 \mathrm{~km} \mathrm{~s}^{-1}$.

[7] If we project the $11.75 \mathrm{~h}$ sequence forward into day 288 , we see that it correctly predicts the timings of two further maxima in the electron flux, labeled $e^{\prime}$ and $f^{\prime}$. The first of these is characterized by a different magnetic signature than hitherto, in which a small increase in the field strength took place in phase with the increase in electron pressure. The electron pressure increased from $\sim 0.0025$ to $\sim 0.0045 \mathrm{nPa}$ at the maximum (potentially indicative of a total plasma pressure increase from $\sim 0.01$ to $\sim 0.015 \mathrm{nPa}$ if the ion pressure is $\sim 2-3$ times the electron pressure as indicated above), while the magnetic pressure increased from $\sim 0.015$ to $\sim 0.02 \mathrm{nPa}$. With reasonable allowance for the ion pressure, therefore, we estimate an increase in total pressure from $\sim 0.025$ to $\sim 0.035 \mathrm{nPa}$ in the oscillation. Similar electron pressures were also reached in the second flux maximum $f^{\prime}$. Between these maxima, however, a brief magnetosheath encounter took place, evidenced by the intense fluxes of low-energy $(\sim 10-$ $100 \mathrm{eV}$ ) electrons, and corresponding changes in the field strength and orientation. That is to say, the magnetopause moved inward across the spacecraft during the low-pressure phase of the oscillation, and then out again as the internal pressure rose once more. These magnetopause crossings occurred at radial distances of $\sim 28.8$ (into the magnetosheath) and $\sim 29.2 \mathrm{R}_{\mathrm{S}}$ (back into the magnetosphere), and are marked by red arrows at the top of the plot. Such observations provide primary evidence for modulation of the magnetopause boundary by internal pressure variations at the (Doppler-shifted) planetary period. Extrapolating the sequence into days 289 and 290 with lines marked $g^{\prime}, h^{\prime}$ and $i^{\prime}$, we find that while no magnetospheric entries occurred around the times of $g^{\prime}$ and $h^{\prime}$, a transient encounter did take place at $i^{\prime}$ near the start of day 290 at a radial distance of $\sim 35 \mathrm{R}_{\mathrm{S}}$. We infer that this encounter was associated with a fall in solar wind dynamic pressure that brought the oscillating boundary once more within range of the spacecraft (see section 3), allowing a transient entry around the time of the internal pressure peak.

[8] We now examine the data from the inbound pass. As mentioned above, the magnetosphere was relatively compressed during Rev 16, such that the spacecraft spent only a short interval inside the magnetosphere prior to periapsis. In this case there is no opportunity to observe a long sequence of variations at around the planetary period. However we note two transient magnetosphere encounters labeled $b$ and $c$ with midpoints at radial distances of $\sim 24.1$ and $\sim 21.4 \mathrm{R}_{\mathrm{S}}$, respectively, which are separated by $\sim 10.25 \mathrm{~h}$ and are followed by a final magnetopause crossing $\sim 8.5 \mathrm{~h}$ later (red arrows). We infer that these are the result of boundary

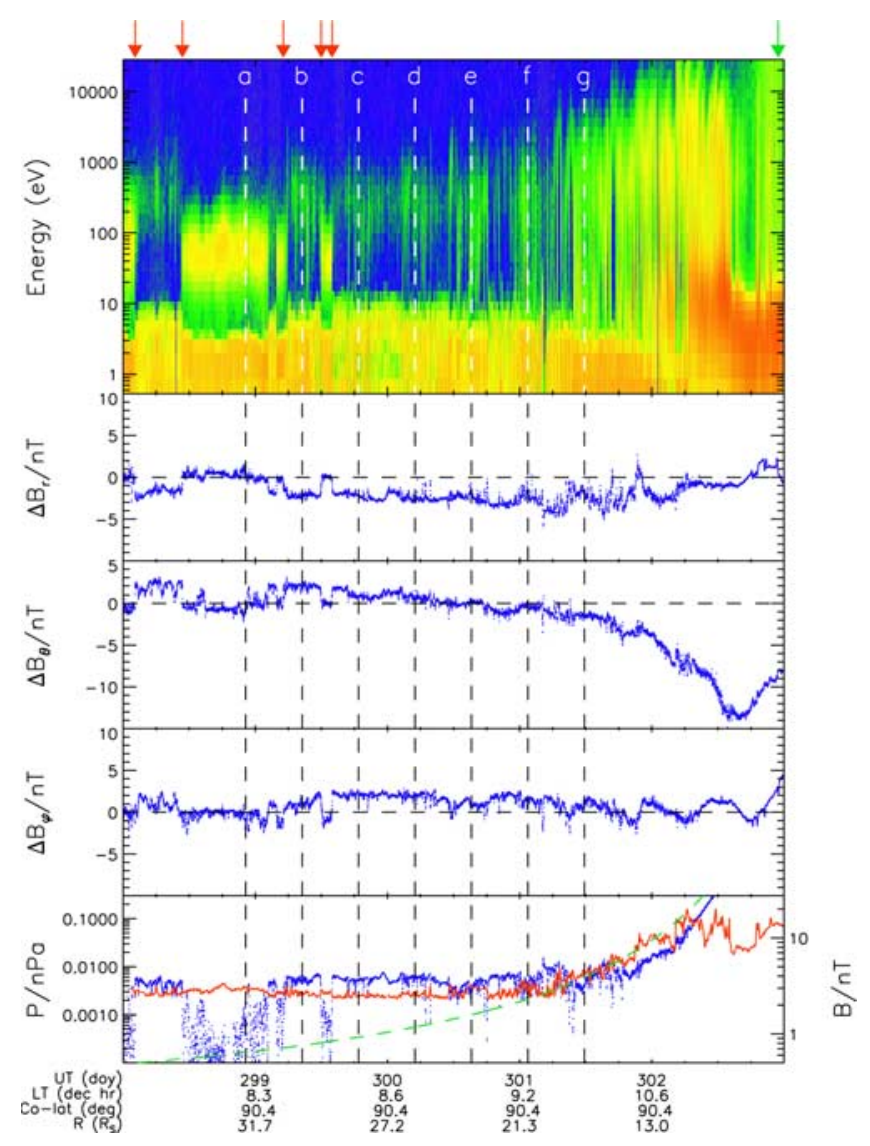

Figure 3. Electron flux spectrogram and magnetic field data from Cassini Rev 17 inbound, days 298-302 inclusive. The electron flux color scale is the same as for Figure 1.

oscillations similar to those observed outbound. The $\sim 10.25 \mathrm{~h}$ period is shorter than the planetary period, however, due to the Doppler shift associated with the spacecraft's radial inward motion. Projecting the $10.25 \mathrm{~h}$ sequence forward in time, we find that $d$ corresponds to a local maximum in the magnetospheric magnetic and electron pressures. Projecting the sequence backward to earlier times, there is no magnetosphere encounter at $a$, but we note a brief excursion into the solar wind between $a$ and $b$, indicated by the interval of low electron flux above $\sim 10 \mathrm{eV}$ and the simultaneous low magnetic field strengths (the bow shock crossings are indicated by blue arrows). This may indicate that the bow shock is also modulated, moving closer to the planet in the low-pressure phase of the oscillation. An earlier transient solar wind encounter late on day 281 does not obey this phasing sequence, however, at least with the period as determined here, perhaps because of additional boundary motion due to changes in solar wind pressure.

[9] Figure 3 shows data for the Rev 17 inbound pass in the same format as Figure 1, for days 298-302 (25-29 Oct) inclusive. The orbit is very similar to Rev 16 and is not shown here. The magnetosphere is now more expanded due to lower solar wind dynamic pressure (see section 3), such that we again observe a series of electron flux maxima inside the magnetosphere, associated with modest increases in electron pressure, of order $\sim 0.001 \mathrm{nPa}$. The period of 
$\sim 10.25 \mathrm{~h}$ is the same as that used (on a less well-constrained basis) for Rev 16 inbound. The main feature of note is the transient magnetosheath entry occupying the low flux region between $b$ and $c$ (at $\sim 29.5 \mathrm{R}_{\mathrm{S}}$ ), again indicating boundary modulation with the planetary period oscillation. Looking to earlier times, no magnetopause encounters are seen near $a$, suggesting that the spacecraft was then beyond the range of the oscillating boundary.

\section{Discussion}

[10] We now consider the implications of the above observations for the amplitude of the boundary oscillations, and for the changes in internal pressure required to produce them. An initial estimate of the amplitude can be obtained by examining the radial range of observed boundary locations on a given pass, this having the nature of a lower limit to the peak-to-trough amplitude assuming an approximately steady solar wind dynamic pressure. To provide a consistent pass-to-pass estimate we have mapped the observed boundary locations to the planet-Sun line using the magnetopause shape given by the model of Arridge et al. [2006], this procedure assuming for simplicity that the variation in oscillation amplitude with local time around the boundary is in rough proportion to the mean boundary distance. Since the Arridge et al. [2006] model is parameterized in terms of the solar wind dynamic pressure $D_{p}$, we also consider the value of this quantity appropriate to the above intervals, validating and quantifying the inferences made in section 2 . In this model the radial distance of the magnetopause boundary, $R_{M P}$, is given by

$$
R_{M P}(\theta)=R_{M P_{S S}}\left(\frac{2}{1+\cos \theta}\right)^{\kappa},
$$

where $R_{M P_{S S}}$ is the subsolar magnetopause radius, and $\theta$ is the angle to the planet-Sun line. Fits to Cassini boundary observations then show that $R_{M P_{S S}} \approx 9.7 D_{p}^{-0.24} \mathrm{R}_{\mathrm{S}}$ and $\kappa \approx$ $0.77-1.46 D_{p}$, where $D_{p}$ is in $\mathrm{nPa}$. If we consider the inbound pass of Rev 16, for example, the mean position of the last three magnetopause crossings on day 283 (at $R_{M P} \approx$ $21.5 \mathrm{R}_{\mathrm{S}}$ and $\theta \approx 45^{\circ}$ ) indicate a dynamic pressure of $\sim 0.05 \mathrm{nPa}$ according to equation (1), with a subsolar radius of $\sim 19 \mathrm{R}_{\mathrm{S}}$, i.e. a relatively compressed magnetosphere as indicated in section 2. A check can also be made by considering the pressure observed inside the magnetopause boundary, $P_{M}$, related to $D_{p}$ by $P_{M} \approx k D_{p} \cos ^{2} \Psi$, where $\mathrm{k} \approx 0.88$ for a high-Mach number flow and $\Psi$ is the angle between the solar wind flow and the boundary normal. Using the Arridge et al. [2006] model to estimate $\Psi\left(\sim 25^{\circ}\right.$ in the present case) then yields the estimate $P_{M} \approx 0.04 \mathrm{nPa}$, which can be seen to be in good agreement with the generally dominant magnetospheric magnetic pressures (corresponding to field strengths $\sim 9-11 \mathrm{nT}$ ) observed inside the inbound magnetopause in Figure 1. The main point here, however, is that if we use the model boundary shape to map the observed boundary radii to the planet-Sun line, the range of subsolar magnetopause distances implied by the observed boundary positions lies between $\sim 17.1$ and $\sim 21.3 \mathrm{R}_{\mathrm{S}}$, such that the subsolar peak-to-trough oscillation amplitude is at least $\sim 4.2 \mathrm{R}_{\mathrm{S}}$. Repeating this analysis for the first three crossings on the outbound pass yields a similarly high dynamic pressure of $\sim 0.07 \mathrm{nPa}$ with a subsolar magnetopause radius of $\sim 18 \mathrm{R}_{\mathrm{S}}$, consistent (for $\Psi \approx 60^{\circ}$ ) with magnetospheric pressures of $\sim 0.02 \mathrm{nPa}$ (field strengths $\sim 6-7 \mathrm{nT}$ ) observed inside the boundary in Figure 1 . The range of magnetopause distances mapped to the subsolar point then varies between $\sim 17.3$ and $\sim 18.5 \mathrm{R}_{\mathrm{S}}$, corresponding to a peak-to-trough oscillation amplitude of at least $\sim 1.2 \mathrm{R}_{\mathrm{S}}$. However, the final outbound magnetopause crossings on Rev 16 at the end of day 289 indicate a lower dynamic pressure of $\sim 0.03 \mathrm{nPa}$ with a more expanded subsolar magnetopause at $\sim 22 \mathrm{R}_{\mathrm{S}}$, consistent (for $\Psi \approx 50^{\circ}$ ) with magnetospheric pressures of $\sim 0.01 \mathrm{nPa}$ (field strengths 4-5 nT) observed inside the boundary. These crossings are therefore not taken to form part of the initial outbound boundary oscillation sequence as previously outlined in section 2. Finally, the final three magnetopause encounters on the Rev 17 inbound pass indicate an even lower dynamic pressure of $\sim 0.015 \mathrm{nPa}$ with a subsolar magnetopause at $\sim 25 \mathrm{R}_{\mathrm{S}}$, consistent (for $\Psi \approx 35^{\circ}$ ) with magnetospheric pressures of $\sim 0.01 \mathrm{nPa}$ observed inside the boundary (Figure 3). The range of magnetopause distances mapped to the subsolar point then varies between $\sim 24.4$ and $\sim 25.4 \mathrm{R}_{\mathrm{S}}$, indicating to a peak-to-trough oscillation amplitude of at least $\sim 1.0 \mathrm{R}_{\mathrm{S}}$.

[11] Although the above estimates individually are subject to uncertainty due both to their nature as lower limits and to the possibility of boundary motion due to changing solar wind dynamic pressure during a particular pass, they collectively suggest a peak-to-trough boundary oscillation amplitude of a few Saturn radii. Another indication may be obtained from the frequency with which such multiple crossings are observed in the Cassini data, the results already discussed suggesting that they are not rare. Although a detailed analysis is beyond the scope of the present paper, initial investigation suggests that multiple boundary crossings (typically three) separated by (Doppler-shifted) planetary-period intervals occur in roughly half of all wellobserved boundary-region passes. For sinusoidal boundary oscillation, an observer moving uniformly across the boundary region has almost equal probability of experiencing either one or three boundary crossings, depending on the relative phasing of the oscillation, if they spend one full oscillation period crossing the region. Our initial results thus suggest that the time typically spent by the spacecraft within the region of oscillations is roughly one planetary period of $\sim 10.75 \mathrm{~h}$. Since the speed of the spacecraft in the boundary region normal to the Arridge et al. [2006] model magnetopause is typically $\sim 3.5 \mathrm{~km} \mathrm{~s}^{-1}$, the implied width of the region (i.e. the peak-to-trough amplitude) is $\sim 2.2 \mathrm{R}_{\mathrm{S}}$ at the spacecraft location, or $\sim 2 \mathrm{R}_{\mathrm{S}}$ on the planet-Sun line, consistent with the above individual estimates. We thus estimate that the peak-to-trough oscillation amplitude is typically $\sim 10 \%$ of the mean radial distance of the boundary. If the typical amplitude is significantly smaller than this then multiple boundary crossings at the planetary period would not be observed at all, while if the amplitude is much larger, then larger numbers of multiple crossings would be the norm, neither of which is the case.

[12] We now consider the physical origins of the boundary oscillations. In line with the discussions of Espinosa et al. [2003b] and Cowley et al. [2006], we suppose that a compressive wave propagates outward into Saturn's mag- 


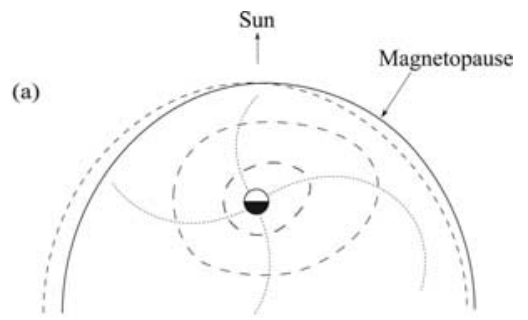

(b)

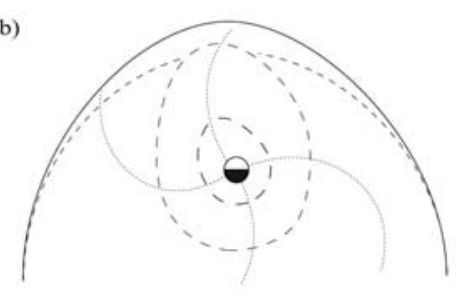

(c)

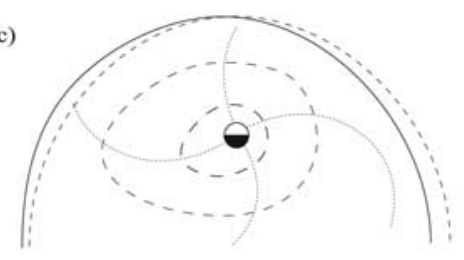

(d)

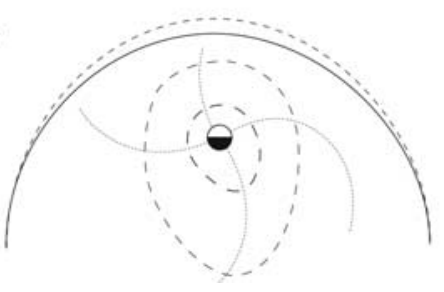

Figure 4. Sketch of Saturn's magnetosphere in a cut through the equatorial plane, shown at intervals of one quarter of the planetary rotation period. The long-dashed lines indicate surfaces of constant plasma pressure, the dotted lines the phase fronts of the wave which correspond to peaks and troughs of the pressure, the solid line the instantaneous magnetopause position, and the short-dashed line its average position.

netosphere from some corotating 'anomaly' at the planet, leading to field and plasma oscillations at the planetary period at any fixed position within the system. This is illustrated in Figure 4, where we sketch the system in the equatorial plane at intervals of one quarter of the planetary period. In these diagrams the long-dashed lines represent surfaces of constant plasma pressure, falling with distance from the planet, while the dotted lines show the phase fronts of the wave corresponding to the peaks and troughs of the pressure, which form a spiral pattern due to the outward propagation of the wave combined with the corotation of the source. As the pressure 'fronts' sweep through the subcorotating plasma in the outer magnetosphere, the magnetopause shown by the outer solid line is displaced outward and inward at the planetary period, as observed. For comparison, the averaged position of the magnetopause is shown by the short-dashed line. Although not shown explicitly, the changing shape of the magnetospheric obstacle will also inevitably lead to planetary-period oscillations in the bow shock position, though only modest evidence for this effect has been found in the data presented here.

[13] We finally make a simple estimate of the magnitude of the internal pressure perturbations required to displace the boundary through typical peak-to-trough amplitudes of $\sim 10 \%$ of the mean position. We first note that for oscillations on the time scale of the planetary period, the boundary position will always be determined by nearequilibrium between the solar wind dynamic pressure and the local pressure inside the boundary. If the boundary were to be displaced from its equilibrium position, the exponential time scale required to move back to equilibrium is $\sim R_{M P} / 3 V_{S W}$, where $V_{S W}$ is the solar wind speed, which is of order $\sim 15 \mathrm{~min}$ at Saturn, much less than the planetary period. Correspondingly, for the amplitudes indicated, the speed of the boundary motion is of order $\sim 5-10 \mathrm{~km} \mathrm{~s}^{-1}$, which is thus negligible compared with the speed of the solar wind. The boundary pressure balance equilibrium condition is taken to be

$$
P_{S W} \approx \frac{2(1+\beta) B_{e q}^{2}}{\mu_{o}}\left(\frac{R_{S}}{R_{M P}}\right)^{6}+\Delta P_{W}(t)
$$

where $P_{S W}$ on the left side is the total pressure of the shocked solar wind outside the boundary, taken to be a constant. The first term on the right side is that of a dipole field compressed by a factor 2 by the boundary currents, and augmented by the background plasma $\beta$ also assumed constant ( $B_{e q}$ is the effective field at the planet's equator, as increased in the outer magnetosphere by the effect of the ring current). The second term on the right side is then the pressure perturbation due to the wave, varying between zero and some maximum value $\Delta P_{W \max }$, taken as a first approximation to be independent of radial distance. With regard to the latter assumption, we note specifically that the amplitude of the wave field is expected to fall with distance much less rapidly than does the background field, such that while wave effects may be negligible compared with the background in the near-planet region, they can readily become comparable with the background at larger distances. If the boundary position when $\Delta P_{W}=0$ is written as $R_{M P 0}$, the value of $\Delta P_{W}$ required to move the boundary to a radius $R_{M P} \geq R_{M P 0}$ is then given by

$$
\begin{aligned}
\Delta P_{W} & \approx \frac{2(1+\beta) B_{e q}^{2}}{\mu_{o}}\left(\frac{R_{S}}{R_{M P 0}}\right)^{6}\left(1-\left(\frac{R_{M P 0}}{R_{M P}}\right)^{6}\right) \\
& \approx P_{S W}\left(1-\left(\frac{R_{M P 0}}{R_{M P}}\right)^{6}\right) .
\end{aligned}
$$

If we put $\left(R_{M P} / R_{M P 0}\right) \approx 1.1$ for a maximum $\sim 10 \%$ increase in the boundary position say, as indicated by the above results, we find $\Delta P_{W \max } \approx 0.44 P_{S W}$, i.e. the increase in pressure must be a significant fraction of the background pressure just inside the magnetosphere, hence also a significant fraction of the solar wind pressure outside. Increases in pressure of such magnitude due to the planetary period oscillations are entirely compatible with the observations presented here. For example, if we consider flux maximum $e^{\prime}$ observed near the boundary on the Rev 16 outbound pass, we estimated in section 2 above (after 
reasonable allowance for the ion pressure) that the total pressure increased from $\sim 0.025$ to $\sim 0.035 \mathrm{nPa}$ in the oscillation, corresponding to an increase of just $\sim 40 \%$, in excellent agreement with the above estimate.

\section{Summary and Conclusions}

[14] The principal results reported in this paper are as follows.

[15] (a) Examination of Cassini magnetic field and plasma data in the outer regions of Saturn's magnetosphere shows that the magnetic oscillations at the planetary period which are ubiquitously present are accompanied by corresponding variations in the electron flux over the energy range from $<10 \mathrm{eV}$ to $>10 \mathrm{keV}$.

[16] (b) Magnetopause boundary oscillations at the planetary period also commonly occur, which are in phase with the plasma pressure variations inside the magnetosphere. Related oscillations in the bow shock position are then also expected, though only marginal evidence for them has been found in the data examined here.

[17] (c) The peak-to-trough amplitude of the magnetopause oscillation on the planet-Sun line is estimated to be typically $\sim 2 \mathrm{R}_{\mathrm{S}}$, corresponding to a $\sim 10 \%$ change in the boundary radius.

[18] (d) The increase in pressure inside the boundary required to produce such motions is estimated to be $\sim 40 \%$ of the background values, and hence $\sim 40 \%$ of the solar wind dynamic pressure. Such increases are compatible with the observations presented here when reasonable allowance is made for the ion pressure.

[19] We propose that these effects are produced by a global-scale compressive wave which propagates outward through the sub-corotating outer magnetospheric plasma, originating from a corotating source in the nearer-planet region. It seems likely that this wave has a profound effect on the plasma dynamics of the outer magnetospheric region, and is the basic causative agent of other oscillating phenomena at Saturn such as modulated kilometric radio emissions [e.g., Kaiser et al., 1980].

[20] Acknowledgments. We thank Michelle Thomsen for providing information on CAPS ion data, and Lin Gilbert for access to CAPS-ELS data. We also thank PPARC for CAPS-ELS and MAG operations support and data analysis, and NASA/JPL for support of Cassini and CAPS. Work at Leicester was supported by PPARC grant PPA/G/O/2003/00013.KEC was supported by a PPARC Postgraduate Studentship, SWHC by a Royal Society Leverhulme Trust Senior Research Fellowship, and MKD by a PPARC Senior Fellowship.

\section{References}

Arridge, C. S., N. Achilleos, M. K. Dougherty, K. K. Khurana, and C. T. Russell (2006), Modeling the size and shape of Saturn's magnetopause with variable dynamic pressure, J. Geophys. Res., 111, A11227, doi:10.1029/2005JA011574.

Cowley, S. W. H., D. M. Wright, E. J. Bunce, A. C. Carter, M. K. Dougherty, G. Giampieri, J. D. Nichols, and T. R. Robinson (2006), Cassini observations of planetary-period magnetic field oscillations in Saturn's magnetosphere: Doppler shifts and phase motion, Geophys. Res. Lett., 33, L07104, doi:10.1029/2005GL025522.

Dougherty, M. K., et al. (2004), The Cassini magnetic field investigation, Space Sci. Rev., 114, 331-383.

Dougherty, M. K., et al. (2005), Cassini magnetometer observations during Saturn orbit insertion, Science, 307, 1266-1270.

Espinosa, S. A., D. J. Southwood, and M. K. Dougherty (2003a), Reanalysis of Saturn's magnetospheric field data view of spin-periodic perturbations, J. Geophys. Res., 108(A2), 1085, doi:10.1029/2001JA005083.

Espinosa, S. A., D. J. Southwood, and M. K. Dougherty (2003b), How can Saturn impose its rotation period in a noncorotating magnetosphere?, J. Geophys. Res., 108(A2), 1086, doi:10.1029/2001JA005084.

Giampieri, G., M. K. Dougherty, E. J. Smith, and C. T. Russell (2006), A regular period for Saturn's magnetic field that may track its internal rotation, Nature, 441, 62-64.

Huddleston, D. E., C. T. Russell, M. G. Kivelson, K. K. Khurana, and L. Bennett (1998), Location and shape of the Jovian magnetopause and bow shock, J. Geophys. Res., 103, 20,075-20,082.

Joy, S. P., M. G. Kivelson, R. J. Walker, K. K. Khurana, C. T. Russell, and T. Ogino (2002), Probabalistic models of the Jovian magnetopause and bow shock locations, J. Geophys. Res., 107(A10), 1309, doi:10.1029/ 2001JA009146.

Kaiser, M. L., M. D. Desch, J. W. Warwick, and J. B. Pearse (1980), Voyager detection of non-thermal radio emission from Saturn, Science, 209, $1238-1240$.

Kivelson, M. G., and D. J. Southwood (2003), First evidence of IMF control of Jovian magnetospheric boundary locations: Cassini and Galileo magnetic field measurements compared, Planet. Space Sci., 51, $891-$ 898.

Shue, J.-H., J. K. Chao, H. C. Fu, C. T. Russell, P. Song, K. K. Khuruna, and H. J. Singer (1997), A new functional form to study the solar wind control of the magnetopause size and shape, J. Geophys. Res., 102, 9497-9511.

Slavin, J. A., E. J. Smith, P. R. Gazis, and J. D. Mihalov (1983), A PioneerVoyager study of the solar wind interaction with Saturn, Geophys. Res. Lett., 10, 9-12.

Young, D. T., et al. (2004), Cassini plasma spectrometer investigation, Space Sci. Rev., 114, 1-112.

N. André, Research and Scientific Support Department, European Space Agency, NL-2200 AG Noordwijk, Netherlands.

D. J. Andrews, K. E. Clarke, S. W. H. Cowley, J. D. Nichols, T. R. Robinson, and D. M. Wright, Department of Physics and Astronomy, University of Leicester, Leicester LE1 7RH, UK. (kec10@ion.le.ac.uk)

A. J. Coates, G. R. Lewis, and H. J. McAndrews, Mullard Space Science Laboratory, University College London, Holmbury St Mary, Dorking RH5 6NT, UK

M. K. Dougherty, Blackett Laboratory, Imperial College London, London SW7 2AZ, UK. 